\title{
Tunable Surface Properties from Sequence-Specific Polypeptoid- Polystyrene Block Copolymer Thin Films
}

\author{
Wendy van Zoelen, ${ }^{\dagger}$ Ronald N. Zuckermann, ${ }^{\dagger}$ and Rachel A. Segalman $*, \dagger,+$ \\ ${ }^{\dagger}$ Department of Chemical and Biomolecular Engineering, University of California, Berkeley, Berkeley, California 94720, United States \\ ${ }^{\ddagger}$ The Molecular Foundry, Lawrence Berkeley National Laboratory, Berkeley, California 94720, United States
}

ABSTRACT: Tunability of polymer surface properties depends crucially on both the chemical composition of the polymer and the physics of the chains (e.g., surface segregation, chain shape, etc.). Polypeptoids, which are nonnatural biomimetic polymers based on an $\mathrm{N}$-substituted glycine backbone, provide a flexible model system in which monomer sequence, chain shape, and self-assembled structure can easily be controlled to understand their influence on surface properties. We demonstrate the influence of the amount and sequence of hydrophobic monomers in a predominantly hydrophilic peptoid chain on the surface

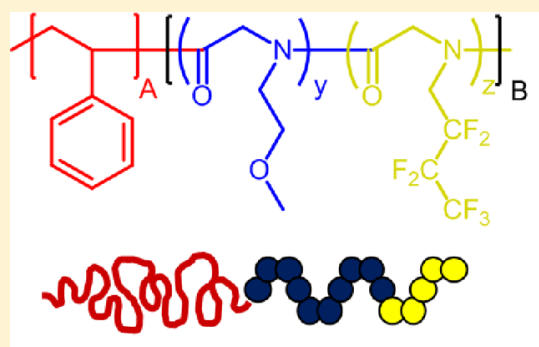

$A \sim 110 \quad y+z=15,30,45$

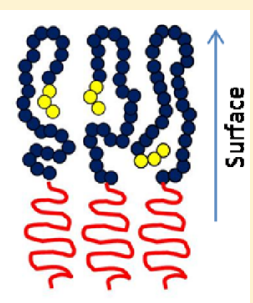

Surface reconstuction after water immersion properties of a hybrid block copolymer, poly(peptoid-b-styrene). Just three fluorinated groups in peptoid sequences consisting of up to 45 hydrophilic monomers in length were needed to lower the surface energy of the peptoid and allow for its maximal surface segregation. Positioning these fluorinated groups in the middle of a chain as opposed to the chain ends resulted in a change in chain conformation at the surface as evidenced by near-edge X-ray absorption fine structure spectroscopy (NEXAFS). Surface reconstruction of polymers containing only three fluorinated monomers occurred within seconds but could be slowed by an order of magnitude when five fluorinated monomers were incorporated.

\section{INTRODUCTION}

For many biological applications, specifically antifouling applications, it is necessary to incorporate multiple functionalities on a single surface. In order to provide a polymeric material with all of these functionalities, multicomponent systems, such as polymer blends, block copolymers, and chainend-functionalized polymers, are often employed. However, the use of multiple functionalities can make the behavior of such a material on the nanometer scale difficult to predict. ${ }^{1}$ In order to visualize this, one can simply think of the complexity of the phase diagram of an $\mathrm{ABC}$ triblock copolymer compared to that of an $\mathrm{AB}$ diblock copolymer. ${ }^{2,3}$ The behavior at a surface can be especially unpredictable, as it involves additional parameters that are not involved with the bulk properties of a material. In particular, surface segregation of the component with the lowest surface free energy can greatly alter the composition and chain conformation at the surface from that in the bulk. The surface behavior becomes even more complex when the polymers are able to reconstruct; a phenomenon that can occur when the processing environment does not correspond to the environment in which the material will be used, as is the case for marine antifouling coatings.

The ban on heavy-metal-containing antifouling coatings has accelerated the need for environmentally friendly alternatives, and many different chemistries have already proven to be effective. Hydrophilic coatings, such as poly(ethylene glycol) and zwitterionic species, have shown good antifouling behavior. ${ }^{4,5}$ Hydrophobic coatings tend to work as fouling release coatings in which foulants can easily be removed from a surface by shear forces. Recently, amphiphilic materials with inherently more complex chemistries have proven to be quite successful as antifouling coatings, although the exact antifouling mechanism is not always clear. ${ }^{4-10}$ In most cases, reconstruction of hydrophobic moieties away from the interface has been observed. Although the extent of reconstruction is difficult to predict or quantify, it is likely to have an effect on the antifouling properties. A more thorough knowledge of the surface segregation behavior and in particular of the necessary requirements to promote or prevent surface reconstruction will aid the development of future amphiphilic antifouling coatings as well as other materials in which tunable surface properties are important.

Sequence-specific polymers are of particular interest for antifouling applications because they allow for the precise control of functional group location relative to a surface and can incorporate multiple functionalities in a modular way. Traditionally, sequence-specific polymers have been available only in quantities insufficient for materials applications due to the challenges and expense inherent to their syntheses. Peptoids are a class of non-natural biomimetic oligomers based on an $\mathrm{N}$-substituted glycine backbone that combine many of the advantageous properties of bulk polymers with

Received: May 15, 2012

Revised: $\quad$ August 7, 2012

Published: August 27, 2012 
those of proteins. Zuckermann and co-workers developed a submonomer synthetic route in which each reaction step occurs at a very high yield such that long, modular, sequence-specific polymers are possible in gram scale batches (Figure 1). ${ }^{11}$

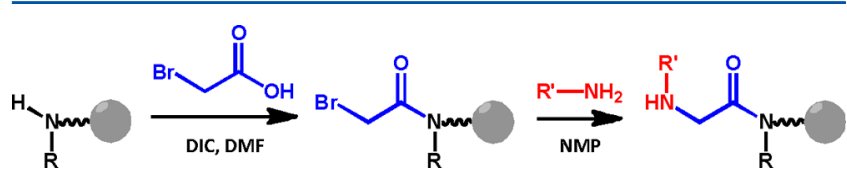

Figure 1. Submonomer solid-state peptoid synthesis scheme. In the first step, the amine functionality on the bead of resin is bromoacetylated, after which the bromine can be displaced with a wide variety of primary amines in the second step. These two steps complete the addition of one monomer to the peptoid chain. This procedure can be repeated to yield a sequence-specific polypeptoid. Because the efficiency of each reaction step is typically $>99 \%$, polymeric lengths are achievable.

Additionally, primary amines are used as submonomers, allowing for the potential inclusion of a broad range of functionalities beyond biomimetics. The majority of early peptoid research focused on peptoids as therapeutic agents. ${ }^{12-16}$ Recent research has indicated peptoids to be highly suitable from a materials science perspective as well. ${ }^{17-21}$ For example, unlike other biopolymers, polypeptoids exhibit enhanced thermal and solution processability due to reduced intermolecular hydrogen bonding and may be thought of as hybrids between conventional polymers and biopolymers. ${ }^{17,22,23}$

In this work, peptoids containing both hydrophilic methoxyethyl side chains and fluorinated aliphatic side chains were used to tune the surface properties of block copolymers of polystyrene and polypeptoids. The polystyrene block acts as a hydrophobic structure-stabilizing agent, whereas the peptoid tunes the surface properties. By controlling the amount and the position of fluorinated peptoid groups and the total peptoid length, the surface display and its reorganization upon immersion in water were investigated. Three fluorinated groups at the end of a peptoid chain up to 45 monomers in length allowed for maximal peptoid surface display, whereby the fluorinated chain ends occupy the free surface. Fluorinated groups in the middle of a chain resulted in loop display at the free surface. Upon immersion in water, reconstruction of surfaces with three fluorinated groups occurred within seconds but could be slowed by an order of magnitude by increasing the number of such groups to five.

\section{EXPERIMENTAL SECTION}

Synthesis of Azide End-Functionalized Polystyrene. Nitroxide-mediated radical polymerization was used to synthesize polystyrene with a chloride end group. The alkoxyamine initiator, $\mathrm{N}$ tert-butyl-O-[1-(4-chloromethylphenyl)ethyl]-N-(2-methyl-1phenylpropyl)hydroxylamine (Cl-BzEt-TIPNO), was synthesized following a previously published procedure. ${ }^{24}$ Styrene monomer (Sigma-Aldrich, $>99 \%$ ) was filtered over basic alumina to remove the inhibitor. Depending on the desired molecular weight, calculated amounts of the filtered styrene and the Cl-BzEt-TIPNO initiator were added to the reaction flask and degassed by three freeze-pump-thaw cycles. The reaction mixture was heated in an oil bath at $115{ }^{\circ} \mathrm{C}$ overnight. The reaction mixture was then precipitated in methanol to give a white powder. The chloride end-functionalized polystyrene $(0.15 \mathrm{mmol})$ was dissolved in DMF $(100 \mathrm{~mL})$, and $25 \mathrm{~mol}$ equiv of sodium azide was added. The reaction mixture was stirred overnight at $60{ }^{\circ} \mathrm{C}$, and afterward, the polymer was precipitated in methanol to yield a white powder.

Polystyrene Characterization. Polystyrene (PS) molecular weights were determined using nuclear magnetic resonance (NMR) spectroscopy, recorded on a Bruker Biospin Avance II $500 \mathrm{MHz}$ high performance NMR spectrometer. Chloroform was used as a solvent.

Peptoid Synthesis. Polypeptoids were synthesized on a custom robotic synthesizer or a commercial Aapptec Apex 396 robotic synthesizer on $100 \mathrm{mg}$ of Rink amide polystyrene resin $(0.6 \mathrm{mmol} / \mathrm{g}$, Novabiochem, San Diego, CA). All primary amine monomers, solvents, and reagents described here were purchased from commercial sources and used without further purification. The synthesis procedure was a modified version of methods previously described. ${ }^{25}$ The Fmoc group on the resin was deprotected by adding $2 \mathrm{~mL}$ of $20 \%(\mathrm{v} / \mathrm{v})$ piperidine/ $N, N$-dimethylformamide (DMF), agitating for $20 \mathrm{~min}$, draining, and washing with DMF. All DMF washes consisted of the addition of $2 \mathrm{~mL}$ of DMF, followed by agitation for $1 \mathrm{~min}$ (repeated five times). An acylation reaction was then performed on the amino resin by the addition of $1.0 \mathrm{~mL}$ of $1.2 \mathrm{M}$ bromoacetic acid in DMF, followed by $0.18 \mathrm{~mL}$ of $\mathrm{N}, \mathrm{N}$-diisopropylcarbodiimide (DIC, 1.15 mmol, neat). The mixture was agitated for $20 \mathrm{~min}$ at room temperature, drained, and washed with DMF. Nucleophilic displacement of the bromide with various primary amines (see Table 1) occurred by a $1.0 \mathrm{~mL}$ addition of the primary amine monomer as a 1.25-1.5 M solution in N-methyl-2-pyrrolidone (NMP), followed by agitation for 60-120 min (depending on peptoid length and type of monomer) at room temperature. The monomer solution was drained from the resin, and the resin was washed with DMF as described above. The acylation and displacement steps were repeated until a polypeptoid of the desired length was synthesized. All reactions were performed at room temperature. All polypeptoids were acetylated on the resin after synthesis. When the last monomer was $\mathrm{N}$-(2methoxyethyl)glycine, acetylation was performed using a mixture (2.0 mL per $100 \mathrm{mg}$ of resin) of $0.4 \mathrm{M}$ acetic anhydride and $0.4 \mathrm{M}$ pyridine in DMF for $30 \mathrm{~min}$, followed by washing with DMF. When the last monomer was heptafluorobutylglycine, acetylation was performed using $1 \mathrm{M}$ acetic anhydride in DMF at $60{ }^{\circ} \mathrm{C}$ for $4 \mathrm{~h}$.

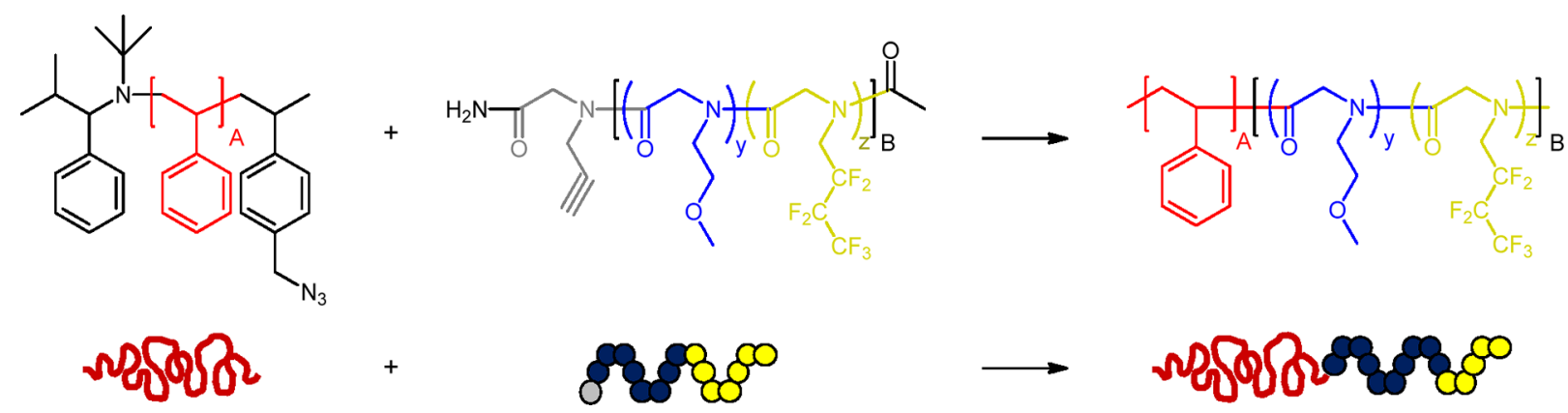

Figure 2. Simplified reaction scheme and corresponding schematic structures for polymers synthesized in this study. A $\sim 110 ; y+z=15$, 30, 45; sequence and ratio $y: z$ in B are varied according to Table 3. 
Table 1. Peptoid Side Chains Used in This Study

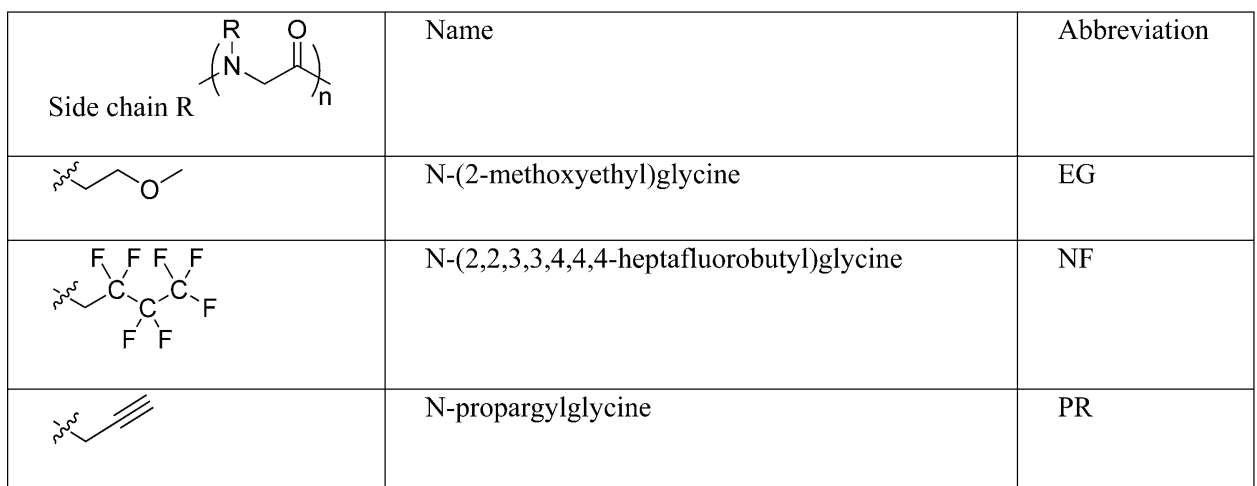

Peptoid chains were cleaved from the resin by addition of $3.0 \mathrm{~mL}$ of cleavage cocktail of $95 \%(\mathrm{v} / \mathrm{v})$ TFA in water for $20 \mathrm{~min}$, which was then evaporated off under a stream of nitrogen gas. Following cleavage, peptoids were dissolved in $4.0 \mathrm{~mL}$ of appropriate acetonitrile/water mixtures and lyophilized twice to obtain a fluffy white powder.

All peptoids (Table 2) were purified by reverse-phase prep HPLC on a Varian ProStar system equipped with a UV-vis single wavelength

\section{Table 2. Peptoids Synthesized in This Study}

\begin{tabular}{|c|c|c|c|}
\hline name & mol wt & obsd mol wt & purity (\%) \\
\hline PR-EG 15 & 1880.1 & 1882.0 & 78 \\
\hline PR-EG $14-\mathrm{NF}$ & 2004.1 & 2006.0 & 95 \\
\hline PR-EG $12-\mathrm{NF}_{3}$ & 2252.0 & 2254.4 & 96 \\
\hline PR-EG $10-\mathrm{NF}_{5}$ & 2500.0 & 2502.9 & 100 \\
\hline PR-EG $-\mathrm{NF}_{7}$ & 2747.8 & 2751.0 & 94 \\
\hline PR-EG 30 & 3607.1 & 3614.3 & 97 \\
\hline PR-EG ${ }_{29}-\mathrm{NF}$ & 3731.0 & 3737.7 & 100 \\
\hline PR-EG ${ }_{27}-\mathrm{NF}_{3}$ & 3979.0 & 3986.0 & 96 \\
\hline PR-EG $25-\mathrm{NF}_{5}$ & 4226.9 & 4231.9 & 92 \\
\hline PR-EG $44-\mathrm{NF}$ & 5458.0 & 5459.5 & 98 \\
\hline PR-EG ${ }_{42}-\mathrm{NF}_{3}$ & 5705.9 & 5707.8 & 94 \\
\hline PR-EG $40-\mathrm{NF}_{5}$ & 5953.8 & 5956.0 & 92 \\
\hline PR-EG ${ }_{21}-\mathrm{NF}_{3}-\mathrm{EG}_{6}$ & 3979.0 & 3985.9 & 99 \\
\hline PR-EG $15-\mathrm{NF}_{3}-\mathrm{EG}_{12}$ & 3979.0 & 3987.0 & 93 \\
\hline PR-EG $-\mathrm{NF}_{3}-\mathrm{EG}_{18}$ & 3979.0 & 3984.3 & 96 \\
\hline PR-EG $-\mathrm{NF}_{3}-\mathrm{EG}_{24}$ & 3979.0 & 3986.5 & 97 \\
\hline
\end{tabular}

detector $(214 \mathrm{~nm})$ and a C4 column (Vydac HPLC Protein C4 column, $10-15 \mu \mathrm{m}, 22 \times 250 \mathrm{~mm}$ ). Appropriate linear gradients with a $60 \%$ increase of solvent $B$ in solvent $A$ over $40 \mathrm{~min}$ at a flow rate of $10 \mathrm{~mL} / \mathrm{min}$ (solvent $\mathrm{A}=0.1 \%$ TFA in water, solvent $\mathrm{B}=0.1 \%$ TFA in acetonitrile) were used.

Peptoid Characterization. The purity of each polypeptoid was determined by analytical reverse-phase HPLC using a C4 column (Vydac 218MS, $5 \mu \mathrm{m}, 2.1 \times 50 \mathrm{~mm}$ ) on a Varian ProStar system (Palo Alto, CA). The column was maintained at $60{ }^{\circ} \mathrm{C}$ while a $30 \mathrm{~min}$ linear gradient of $5-95 \%$ solvent $\mathrm{B}$ in solvent $\mathrm{A}$ was used (solvent $\mathrm{A}=0.1 \%$ TFA in water, solvent $B=0.1 \%$ TFA in acetonitrile).

The molecular weight was determined using an Applied Biosystems MALDI TOF/TOF Analyzer 4800 with a 1:1 (v/v) mixture of peptoid $(2 \mathrm{mg} / \mathrm{mL}$ in $1: 1$ acetonitrile/water) and 1,8,9-dianthracenetriol (10 $\mathrm{mg} / \mathrm{mL}$ in tetrahydrofuran).

Block Copolymer Synthesis. The block copolymers (Table 3) were synthesized by click chemistry, using a method previously employed by Rosales et al., ${ }^{26}$ which is a modification of the procedure described by Holub et al. ${ }^{27}$ The azide end-functionalized polystyrene $(1.8 \mathrm{mM})$ was allowed to react with 2 equiv of the alkynefunctionalized polypeptoid $(3.7 \mathrm{mM}), 5$ equiv of $\mathrm{Cu}(\mathrm{I}) \mathrm{I}(9.5 \mathrm{mM})$, 6 equiv of ascorbic acid $(10.8 \mathrm{mM})$, and 10 equiv of DIPEA $(18 \mathrm{mM})$ in DMF in a $20 \mathrm{~mL}$ scintillation vial. The vial was purged with nitrogen, capped, and sealed with Parafilm. To ensure that the reactants were dissolved, the vial was placed into a bath sonicator (Fisher Scientific, Solid State Ultrasonic FS-9) for $10 \mathrm{~min}$. It was then shaken at room temperature overnight. Upon completion of the reaction, 15- and 30-mer peptoid block copolymers were precipitated in a 10:90 acetonitrile/water mixture. The 45-mer peptoid block copolymers did not precipitate readily. These reaction mixtures were concentrated in a Genevac evaporator, redissolved in a small amount of THF, and added to a 20:80 acetonitrile/water mixture. These mixtures were concentrated using Amicon Ultra centrifugal filter units (10 000 cutoff $M_{\mathrm{w}}$, Millipore) and resuspended in 20:80 acetonitrile/ water. This procedure was repeated twice. All concentrated block copolymer solutions were lyophilized from 50:50 acetonitrile/water mixtures. Residual copper ions were removed by stirring a THF solution of the block copolymers in a 10-fold excess of Dowex ionexchange resin (Aldrich) overnight. Yields were typically in the 70$90 \%$ range.

Block Copolymer Characterization. In order to determine molecular weights and polydispersities, gel permeation chromatography (GPC) was performed on a Viscotek TDA 302 SEC with a set of four Styrogel HR columns (one HR2, two HR3, and one HR4 column). Refractive index was used for molecular weight determination with the use of polystyrene calibration standards (Polymer Laboratories). Using a flow rate of $1 \mathrm{~mL} / \mathrm{min}$, the mobile phase was THF at $30{ }^{\circ} \mathrm{C}$ (see Figure 3 for typical GPC traces).

Differential scanning calorimetry (DSC) was carried out using a Thermal Advantage Q20 calorimeter equipped with a refrigerated cooling system (both TA Instruments, New Castle, DE). 2-8 mg of lyophilized peptoid powder or block copolymer powder was hermetically sealed into an aluminum pan, and each sample was taken through three temperature cycles whereby samples were equilibrated at $-40{ }^{\circ} \mathrm{C}$, heated to $150{ }^{\circ} \mathrm{C}\left(180{ }^{\circ} \mathrm{C}\right.$ for $\left.\mathrm{PR}-\mathrm{EG}_{8}-\mathrm{NF}_{7}\right)$ with a rate of $10{ }^{\circ} \mathrm{C} / \mathrm{min}$, and cooled again to $-40{ }^{\circ} \mathrm{C}$ with the same rate. The first cycle from each sample was discarded in order to erase the thermal history.

Thin Film Preparation and Characterization. Films with a thickness of $\sim 60 \mathrm{~nm}$ were spin-coated at $2000 \mathrm{rpm}$ from $1 \%(\mathrm{w} / \mathrm{v})$ block copolymer solutions in methyl ethyl ketone (MEK) onto plasma-cleaned silicon wafers. All films were vacuum-annealed for at least $16 \mathrm{~h}$ at $130{ }^{\circ} \mathrm{C}\left(175{ }^{\circ} \mathrm{C}\right.$ for $\left.\mathrm{PS}_{11 \mathrm{~K}}-b-\left(\mathrm{EG}_{8}-\mathrm{NF}_{7}\right)\right)$.

Near-edge X-ray absorption fine structure (NEXAFS) experiments were conducted on the U7A NIST/Dow materials characterization end-station at the National Synchrotron Light Source at Brookhaven National Laboratory (BNL). The general NEXAFS principles and a description of the beamline at BNL have been previously reported. ${ }^{28-30}$ The X-ray beam was elliptically polarized with a polarization factor of 0.85 and the electric field vector dominantly in the plane of the storage ring. The photon flux was $\sim 1 \times 10^{11}$ photons per second at a typical storage ring current of $750 \mathrm{~mA}$. A spherical grating monochromator was used to obtain monochromatic soft Xrays at an energy resolution of $0.2 \mathrm{eV}$. The $\mathrm{C} 1 \mathrm{~s}$ NEXAFS spectra were acquired for incident photon energies in the range of $270-320 \mathrm{eV}$. The partial electron yield (PEY) signal was collected using a 
Table 3. Block Copolymers Synthesized in This Study

\begin{tabular}{|c|c|c|c|c|c|}
\hline $\mathrm{Nr}$. & Name & $\mathrm{A}$ & $x+y$ & $\mathrm{y}$ & Schematic representation \\
\hline 1 & $\mathrm{PS}_{11 \mathrm{k}}-b-\left(\mathrm{EG}_{15}\right)$ & $11 \mathrm{k}$ & 15 & 0 & \\
\hline 2 & $\mathrm{PS}_{11 \mathrm{k}}-b-\left(\mathrm{EG}_{14}-\mathrm{NF}\right)$ & $11 \mathrm{k}$ & 15 & 1 & \\
\hline 3 & $\mathrm{PS}_{11 \mathrm{k}}-b-\left(\mathrm{EG}_{12}-\mathrm{NF}_{3}\right)$ & $11 \mathrm{k}$ & 15 & 3 & \\
\hline 4 & $\mathrm{PS}_{11 \mathrm{k}}-b-\left(\mathrm{EG}_{10}-\mathrm{NF}_{5}\right)$ & $11 \mathrm{k}$ & 15 & 5 & ns \\
\hline 5 & $\mathrm{PS}_{11 \mathrm{k}}-b-\left(\mathrm{EG}_{8}-\mathrm{NF}_{7}\right)$ & $11 \mathrm{k}$ & 15 & 7 & \\
\hline 6 & $\mathrm{PS}_{11 \mathrm{k}}-b-\left(\mathrm{EG}_{30}\right)$ & $11 \mathrm{k}$ & 30 & 0 & \\
\hline 7 & $\mathrm{PS}_{11 \mathrm{k}}-b-\left(\mathrm{EG}_{29}-\mathrm{NF}\right)$ & $11 \mathrm{k}$ & 30 & 1 & \\
\hline 8 & $\mathrm{PS}_{11 \mathrm{k}}-b-\left(\mathrm{EG}_{27}-\mathrm{NF}_{3}\right)$ & $11 \mathrm{k}$ & 30 & 3 & \\
\hline 9 & $\mathrm{PS}_{11 \mathrm{k}}-b-\left(\mathrm{EG}_{25}-\mathrm{NF}_{5}\right)$ & $11 \mathrm{k}$ & 30 & 5 & \\
\hline 10 & $\mathrm{PS}_{11 \mathrm{k}}-b-\left(\mathrm{EG}_{44}-\mathrm{NF}\right)$ & $11 \mathrm{k}$ & 45 & 1 & \\
\hline 11 & $\mathrm{PS}_{11 \mathrm{k}}-b-\left(\mathrm{EG}_{42}-\mathrm{NF}_{3}\right)$ & $11 \mathrm{k}$ & 45 & 3 & \\
\hline 12 & $\mathrm{PS}_{11 \mathrm{k}}-b-\left(\mathrm{EG}_{40}-\mathrm{NF}_{5}\right)$ & $11 \mathrm{k}$ & 45 & 5 & \\
\hline 13 & $\mathrm{PS}_{13.7 \mathrm{k}}-b-\left(\mathrm{EG}_{30}\right)$ & $13.7 \mathrm{k}$ & 30 & 3 & \\
\hline 14 & $\mathrm{PS}_{13.7 \mathrm{k}}-b-\left(\mathrm{EG}_{21}-\mathrm{NF}_{3}-\mathrm{EG}_{6}\right)$ & $13.7 \mathrm{k}$ & 30 & 3 & \\
\hline 15 & $\mathrm{PS}_{13.7 \mathrm{k}}-b-\left(\mathrm{EG}_{15}-\mathrm{NF}_{3}-\mathrm{EG}_{12}\right)$ & $13.7 \mathrm{k}$ & 30 & 3 & $\Omega 8$ \\
\hline 16 & $\mathrm{PS}_{13.7 \mathrm{k}}-b-\left(\mathrm{EG}_{9}-\mathrm{NF}_{3}-\mathrm{EG}_{18}\right)$ & $13.7 \mathrm{k}$ & 30 & 3 & 00 \\
\hline 17 & $\mathrm{PS}_{13.7 \mathrm{k}}-b-\left(\mathrm{EG}_{3}-\mathrm{NF}_{3}-\mathrm{EG}_{24}\right)$ & $13.7 \mathrm{k}$ & 30 & 3 & \\
\hline
\end{tabular}

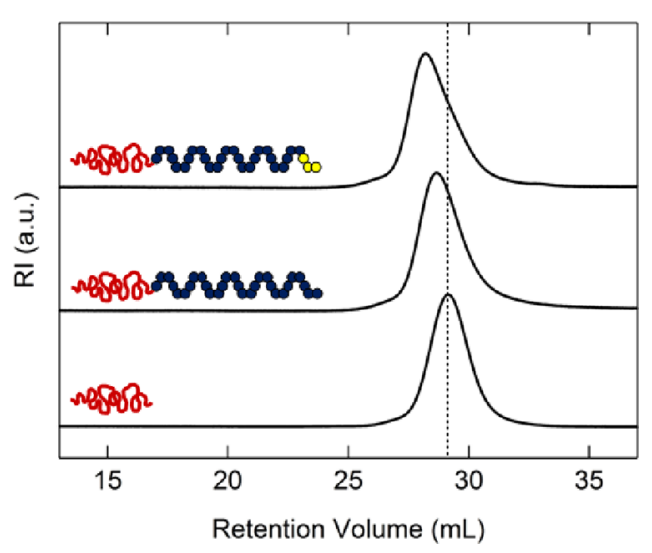

Figure 3. Typical GPC traces for fluorinated and unfluorinated polymers.

channeltron electron multiplier with an adjustable entrance grid bias (EGB), which was set to $-150 \mathrm{~V}$. The channeltron PEY detector was positioned at an angle of $36^{\circ}$ relative to the incoming X-ray beam in the plane defined by the sample normal and the X-ray beam and at an angle of $35^{\circ}$ out of that plane. The incidence angle of the X-ray beam, which was measured from the sample surface, was varied between $20^{\circ}$ and $120^{\circ}$. The PEY C 1 s spectra were normalized by subtracting a linear pre-edge baseline and setting the edge jump to unity at 320 $\mathrm{eV} .{ }^{31}$ The photon energy was calibrated by adjusting the peak position of the lowest $\pi^{*}$ phenyl resonance from polystyrene to $285.5 \mathrm{eV}^{32}$

Tapping mode atomic force microscopy (AFM) measurements were conducted on a Digital Instruments MultiMode AFM equipped with a Nanoscope IIIa controller. Silicon cantilevers (Nanosensors, type PPP-NCL-50, force constant $21-98 \mathrm{~N} / \mathrm{m}$, resonance frequency $146-236 \mathrm{kHz}$ ) were used.

Contact angles were determined using a ramé-hart Model 290 standard automated goniometer equipped with an automated tilting base. Static angles were determined from a $2 \mu \mathrm{L}$ drop of Milli- $\mathrm{Q}$ water. For dynamic measurements, the base was tilted from 0 to $90^{\circ}$ with a speed of $2^{\circ} / \mathrm{s}$ within seconds after depositing a $2 \mu \mathrm{L}$-sized drop on the surface, while recording the advancing and receding contact angles. Each static and dynamic measurement was repeated at least three times at different locations on the surface.

\section{RESULTS AND DISCUSSION}

Peptoid Homopolymer Thermal Properties. Previous studies have shown that the crystallinity of peptoids is tunable. By inserting noncrystalline defects in a chain of crystalline monomers, the melting temperature can be decreased in a controlled fashion. ${ }^{17}$ Peptoids also have the ability to form highly crystalline 2D nanostructures. ${ }^{19,33}$ However, peptoid 
homopolymers with $\mathrm{N}$-2-methoxyethyl side chains are not crystalline and have a $T_{\mathrm{g}}$ below room temperature. As crystalline heptafluorobutyl defects are incorporated into the chain, this $T_{\mathrm{g}}$ increases in a similar fashion as decreasing the melting temperature of a crystalline chain through the incorporation of noncrystalline defects. Figure 4 shows the DSC thermograms of a 16-mer peptoid containing an increasing fraction of heptafluorobutyl side chains.

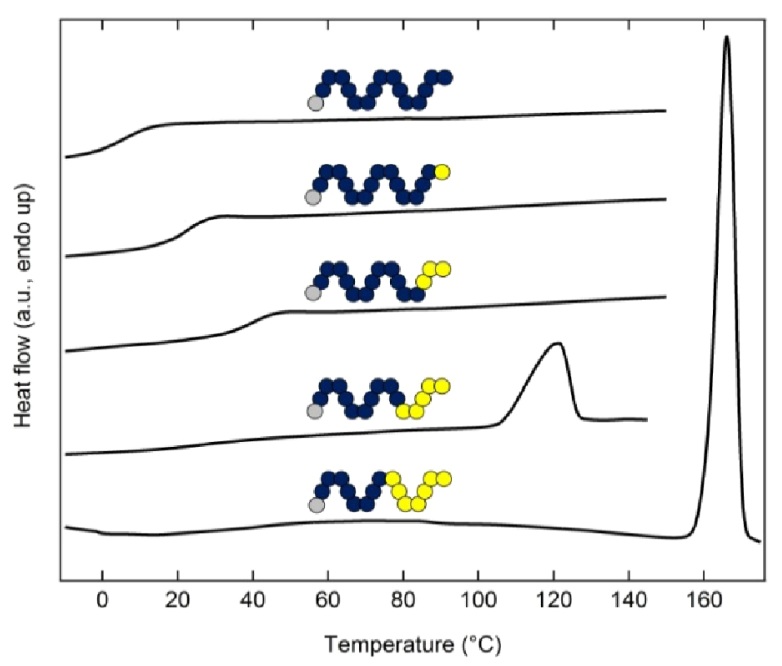

Figure 4. DSC of 16-mer peptoids containing one propargyl side chain (gray circles, later used for click chemistry with azide-functionalized polystyrene) and varying amounts of $\mathrm{N}$-2-methoxyethyl (blue circles) and heptafluorobutyl (yellow circles) side chains. See Table 4 for numerical data.

The $T_{\mathrm{g}}$ increases upon the addition of the fluorinated monomers, but for more than three of such monomers, fluorous interactions result in a melting transition. Seven fluorinated monomers lead to a sharper melting transition of higher enthalpy at higher temperature. It is likely that when five fluorinated groups are incorporated into the peptoid chain, there is no clear separation between the different types of monomers, and the methoxyethyl units are included in the poorly ordered crystalline structure. For seven fluorinated groups, the crystalline structure is likely more ordered and contains less of the methoxyethyl units.

When the number of fluorinated groups in the peptoid chain is held constant at five but the number of methoxyethyl units is increased to a total peptoid length of either 31 or 46 monomers, the peptoid exhibits a $T_{\mathrm{g}}$ at $35{ }^{\circ} \mathrm{C}$ (Figure $4 \mathrm{a}$ ). The $T_{\mathrm{g}}$ is barely visible for the 16-mer, likely due to the short length of the noncrystalline block and a large fraction of the methoxyethyl residues being incorporated into the poorly ordered crystalline structure. The melting temperature of the 31 -mer is slightly higher than that of the 16-mer, and the enthalpy is also higher, indicating a more ordered crystalline structure. While the melting temperature of the 46-mer is the same as that of the 31-mer, the enthalpy is relatively low due to the increased volume fraction of the noncrystalline monomers.

Interestingly, when the crystalline peptoids are coupled to polystyrene, the melting temperature varies with peptoid length, especially for the longest 45-mer peptoid block copolymer the melting transition is significantly lowered. This likely is a result of interactions between PS and the peptoid. The explanation of this effect will require more extensive research into the bulk properties of the system, which is beyond the scope of this paper. For now, Figure $5 b$ serves to indicate that peptoids with five fluorinated groups remain crystalline even when a PS block is attached.
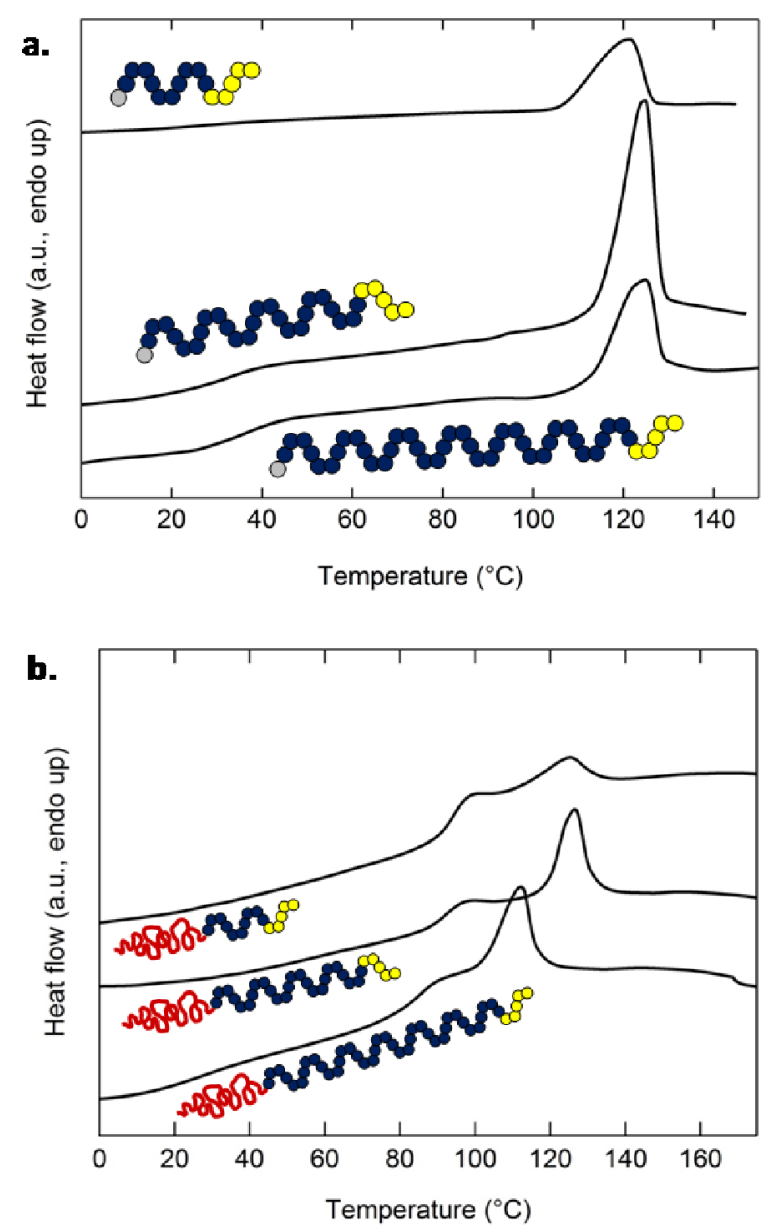

Figure 5. DSC of (a) 16-mer, 31-mer, and 46-mer peptoids containing one propargyl side chain (gray circles, later used for click chemistry with azide-functionalized polystyrene), five heptafluorobutyl (yellow circles) side chains, and 10, 25, or 40 methoxyethyl (blue circles) side chains and (b) their block copolymers with PS. See Table 4 for numerical data.

Although the DSC results do not show the behavior of these molecules in a liquid environment, they indicate that the interaction between the fluorinated groups is strong, and this interaction certainly influences the (surface reconstruction) behavior of these molecules in water, as will be discussed below.

Peptoid NEXAFS. NEXAFS (Figure 6) has been used as the main surface characterization tool in this research. By varying the incidence angle of the polarized X-ray beam $(\theta)$, information about bond orientation can be obtained, whereas variation of the angle between the detector and the surface normal $(\varphi)$ results in different measurement depth. As a result of the setup of beamline $\mathrm{U} 7 \mathrm{~A}$ at $\mathrm{BNL}, \varphi$ and $\theta$ are correlated as $\varphi=\theta-54$. The probed surface depth at a given grid bias is equal to $d \cdot \cos \varphi$. Therefore, at angles of $\theta$ and $180-\theta$, e.g., 60 and 120 , the orientational information is comparable, whereas the probed surface depth differs. For the values of $\theta$ used in this study, 30,60, 90, and 120, the probed surface depths are 0.91 , $0.99,0.81$, and $0.41 \cdot d$, respectively. This means that angles of $\theta$ $<90^{\circ}$ mainly provide orientational information at roughly the 
Table 4. $T_{g}, \Delta C_{p}, T_{\mathrm{m}}$ and $\Delta H$ Values for DSC Traces in Figures 4 and 5

\begin{tabular}{|c|c|c|c|c|}
\hline sample & $T_{\mathrm{g}}\left({ }^{\circ} \mathrm{C}\right)$ & $\Delta C_{p}\left(\mathrm{~J} /\left(\mathrm{g}^{\circ} \mathrm{C}\right)\right)$ & $T_{\mathrm{m}}\left({ }^{\circ} \mathrm{C}\right)$ & $\Delta H(\mathrm{~J} / \mathrm{g})$ \\
\hline PR-EG 15 & 9.09 & 0.37 & & \\
\hline PR-EG ${ }_{14}-\mathrm{NF}$ & 22.72 & 0.40 & & \\
\hline PR-EG $12-\mathrm{NF}_{3}$ & 40.64 & 0.32 & & \\
\hline PR-EG $10-\mathrm{NF}_{5}$ & & & 121.20 & 21.76 \\
\hline 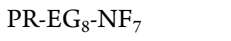 & & & 167.83 & 33.87 \\
\hline PR-EG $25-\mathrm{NF}_{5}$ & 30.87 & 0.26 & 124.06 & 17.22 \\
\hline PR-EG ${ }_{40}-\mathrm{NF}_{5}$ & 36.56 & 0.23 & 124.64 & 8.91 \\
\hline $\mathrm{PS}_{11 \mathrm{k}}-b-\left(\mathrm{EG}_{12}-\mathrm{NF}_{3}\right)$ & 94.36 & 0.20 & 125.06 & 1.32 \\
\hline $\mathrm{PS}_{11 \mathrm{k}}-b-\left(\mathrm{EG}_{25}-\mathrm{NF}_{5}\right)$ & 92.04 & 0.11 & 126.58 & 3.09 \\
\hline $\mathrm{PS}_{11 \mathrm{k}}-b-\left(\mathrm{EG}_{40}-\mathrm{NF}_{5}\right)$ & 85.50 & 0.15 & 112.18 & 2.53 \\
\hline
\end{tabular}

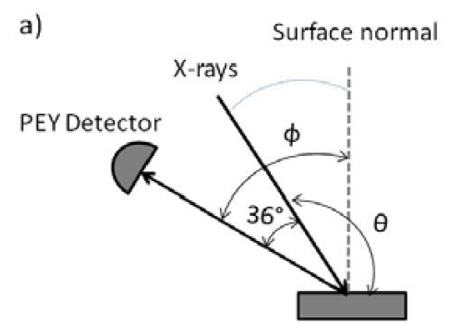

b)

c)

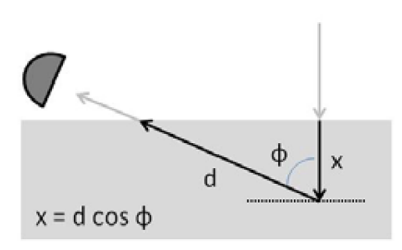

d)

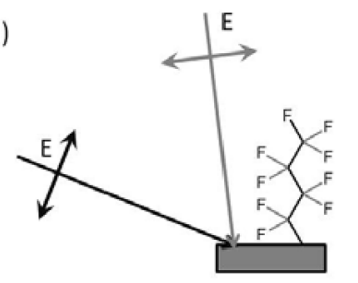

e)

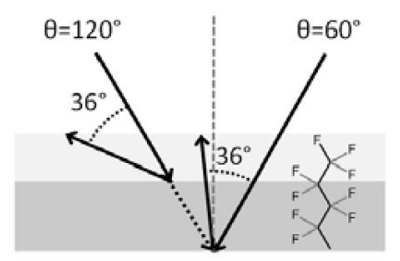

Figure 6. (a) Typical NEXAFS setup at high $\theta$ values, resulting in more surface sensitive measurements. (b) Typical setup at low $\theta$ to probe to greater surface depths. (c) Angle-dependent surface sensitivity. For higher $\varphi$, the probed surface depth, $x$, becomes smaller at constant electron escape depth, $d$. (d) Illustration of the effect of the X-ray beam orientation on bond sensitivity. For the black line, the polarization of the beam is in line with most of the $\mathrm{C}-\mathrm{C} \sigma$ bonding orbitals, whereas for the gray line, the polarization is in line with the $\mathrm{C}-\mathrm{F} \sigma$ bonding orbitals. Note that $\pi$ orbitals are directed orthogonally to a bond, inversing the effects. (e) Illustration of the difference between angles of $\theta$ and $180-\theta$. Both have the same bond orientation sensitivity, but probe different surface depths due to different angles of the detector with the surface normal as depicted in (c).

same surface depth (within $20 \%$ error), whereas the technique becomes increasingly surface sensitive for $\theta>90^{\circ}$.

Figure 7 provides a NEXAFS spectrum for a $\theta$ angle of $60^{\circ}$ for a peptoid containing the fluorinated, methoxyethyl, and propargyl side chains that were described above (Table 1). The angle is close to the "magic angle" of $54^{\circ}$ at which any net bond orientation does not influence the spectrum. ${ }^{34}$ The $\mathrm{C} 1 \mathrm{~s}$ spectrum was fit using a series of (asymmetric) Gaussian peaks

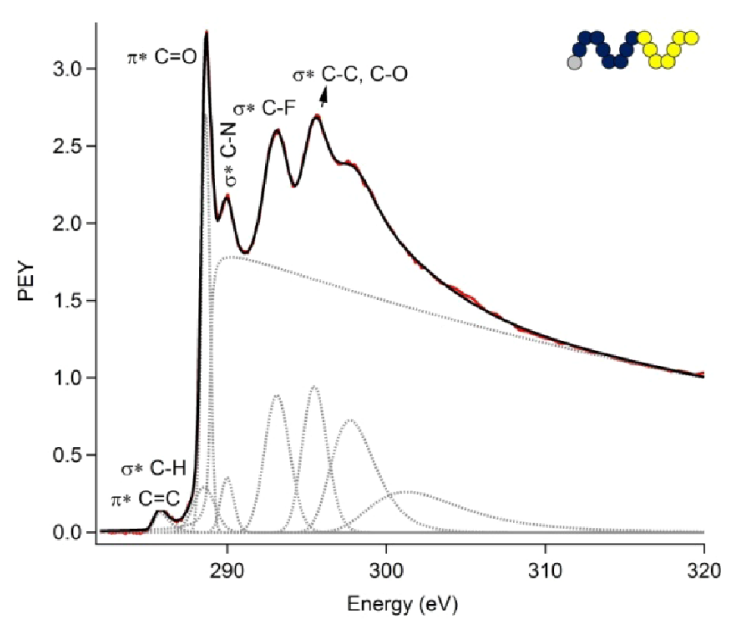

Figure 7. C 1s NEXAFS spectrum and least-squares curve fit of PR$\mathrm{EG}_{8}-\mathrm{NF}_{7}\left(\chi^{2}=0.053\right)$.

for resonances corresponding to bound-state transitions and an arctangent step function for the continuum step. ${ }^{34}$ Peak assignment was based on the calibrated spectra of polystyrene and other polymers. ${ }^{10,34}$ The propargyl group causes a minor $\mathrm{C}$ $1 \mathrm{~s} \rightarrow \pi^{*} \mathrm{C} \equiv \mathrm{C}$ transition at $285.5 \mathrm{eV}$. This is followed by a strong $\mathrm{C} 1 \mathrm{~s} \rightarrow \pi^{*} \mathrm{C}=\mathrm{O}$ transition at $288.6 \mathrm{eV}$. Other characteristic features are the $\sigma^{*}{ }_{\mathrm{C}-\mathrm{N}}, \sigma^{*}{ }_{\mathrm{C}-\mathrm{F}}$, and $\sigma^{*}{ }_{\mathrm{C}-\mathrm{C}}$ and $\sigma^{*}{ }_{\mathrm{C}-\mathrm{O}}$ transitions at 290.0, 293.1, and $295.4 \mathrm{eV}$, respectively. The continuum step occurs at $288.9 \mathrm{eV}$.

PS-Peptoid Block Copolymers. To probe the efficacy of fluorinated monomers in influencing the surface presentation of the peptoid block, a series of polystyrene-peptoid block copolymers with various peptoid lengths and various amounts of fluorinated monomers was synthesized. The molecular weight of the polystyrene block was kept constant (Figure 2 and Table 3, entries 1-12). While such block copolymers can readily self-assemble for appropriate volume fractions in the $\mathrm{bulk}^{26}$ we were solely interested in the surface properties of these molecules.

The surface behavior of these molecules was probed with NEXAFS, using a grid bias of $-150 \mathrm{~V}$. It is difficult to determine the probing depth of the measurement because that depth depends on the sample material; however, at the same grid bias, Genzer et al. and Sohn et al. both determined the escape depth of several fluorinated compounds to be less than 3 $\mathrm{nm}$ ( 2.43 and $1.95 \mathrm{~nm}$, respectively)..$^{30,35}$

Figure 8 shows that increasing the number of fluorinated groups in PS-b-peptoid block copolymers with a 30-mer peptoid (not counting the propargyl monomer used for clicking the peptoid to the azide-functionalized polystyrene) results in increased surface segregation of the peptoid block. When no fluorinated groups are present and the peptoid only contains methoxyethyl side chains, the spectrum looks very much like that of pure PS, indicating that PS segregates to the top surface. As expected, the hydrophilic peptoid has a higher surface free energy than PS. When only one fluorinated group is present, the appearance of the spectrum changes slightly, and a small shoulder near $288.6 \mathrm{eV}$ emerges. This shoulder can be attributed to the strongest transition in the peptoid, the $1 \mathrm{~s} \rightarrow$ $\pi^{*}{ }_{\mathrm{C}=\mathrm{O}}$ transition (see Figure 7). Furthermore, the $\pi^{*}{ }_{\mathrm{C}=\mathrm{C}}$ transition at $285.5 \mathrm{eV}$, which can solely be attributed to PS, slightly decreases. These results indicate that a small percentage of the peptoid is now present at or near the surface, but most of 


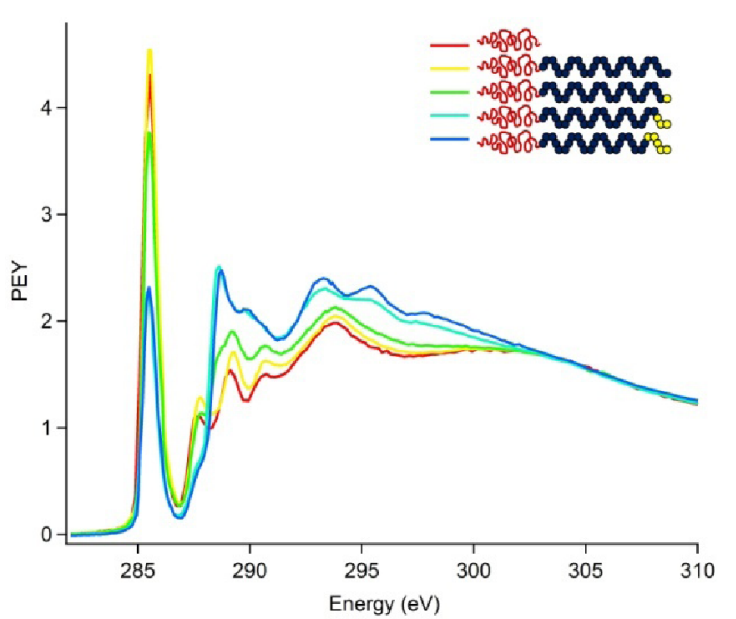

Figure 8. C 1s NEXAFS spectra of block copolymers of PS with 30mer peptoids with various numbers of fluorinated groups $\left(\theta=60^{\circ}\right.$ for all spectra).

the surface still consists of PS. When three fluorinated groups are present, the shape of the spectrum changes considerably, and the typical triple peak structure between 293 and $298 \mathrm{eV}$ corresponding to the $\mathrm{C}_{n} \mathrm{~F}_{2 n+1}$ carbon-fluorine helix is now clearly visible. ${ }^{36}$ Furthermore, the $\pi^{*} \mathrm{C}=\mathrm{C}$ transition decreases considerably. These results suggest substantial movement of the fluorinated peptoid blocks to the surface due to a decreased surface energy caused by the fluorinated groups. However, adding more fluorinated groups does not further increase the presence of the peptoid block at the surface, as illustrated by the spectrum of PS- $b-\left(E_{25}-\mathrm{NF}_{5}\right)$ (dark blue line). This spectrum nearly overlaps with that of PS- $b-\left(E_{27}-N_{3}\right)$, though there is some difference in the signal corresponding to the $1 \mathrm{~s} \rightarrow$ $\sigma^{*}{ }_{\mathrm{C}-\mathrm{F}}, \sigma^{*}{ }_{\mathrm{C}-\mathrm{O}}$, and $\sigma^{*}{ }_{\mathrm{C}-\mathrm{C}}$ transitions. Because of the higher fraction of fluorinated groups, the $\sigma^{*}{ }_{\mathrm{C}-\mathrm{F}}$ peak increases, but the $\pi^{*}{ }_{\mathrm{C}=\mathrm{C}}$ peak does not change. This indicates that an identical amount of PS is at the surface and that surface presentation of the peptoid maximizes for three fluorinated groups in the chain. For a more grazing incidence angle of the X-ray beam, the NEXAFS technique is even more surface sensitive. A decreased $\pi^{*}{ }_{\mathrm{C}=\mathrm{C}}$ peak at this angle, as well as the increased signals above $288 \mathrm{eV}$, corroborate the assumption of preferential surface segregation of the peptoid (Figure 9a).

Block copolymers of PS with a longer 45-mer peptoid (Figure 9b) exhibit similar NEXAFS behavior. However, the $\pi^{*}{ }_{\mathrm{C}=\mathrm{O}}$ shoulder for PS- $b-\left(\mathrm{EG}_{44}-\mathrm{NF}\right)$ is absent, indicating substantially less peptoid occupation of the surface when only one fluorinated group is incorporated in the chain. It is likely this behavior is due to a smaller volume fraction of fluorinated monomer compared to the hydrophilic residues of the peptoid ( 1 out of 45); the amount of fluorinated monomer present might not be enough to promote more of the peptoid to the surface. The 15-mer peptoid block copolymers exhibit roughly the same behavior, although changes in the spectra are more difficult to observe (Figure 9c). This difficulty might be explained by the low fraction of total peptoid to PS, which decreases the overall peptoid NEXAFS signal.

For all peptoid-PS block copolymers, the surface presentation of peptoid maximizes when the peptoid contains three fluorinated groups. The difference between all spectra is the height of the $\pi^{*}{ }_{C}=\mathrm{C}$ peak that corresponds to PS. The area of this peak decreases as the peptoid length increases, indicating a higher amount of peptoid populating the surface for a longer peptoid block. Figure 10 schematically depicts the surface presentation behavior.

The surface schematic depicts sharp boundaries between the peptoid phase and the PS. In reality, the boundaries between the different surface layers are less well-defined due to mixing of both blocks and chain conformation constraints. All peptoid blocks are relatively short compared to the PS block, and complete surface segregation of the peptoid would require considerable unfavorable stretching of the PS block. In fact, we can estimate the extent of phase mixing at the surface. We can integrate the $\pi^{*}{ }_{\mathrm{C}=\mathrm{C}}$ peak to measure the amount of PS that is detected at a certain angle $\theta$, and define a relative degree of mixing $A$ :

$$
A=\frac{d_{120} y_{60}}{d_{60} y_{120}}
$$
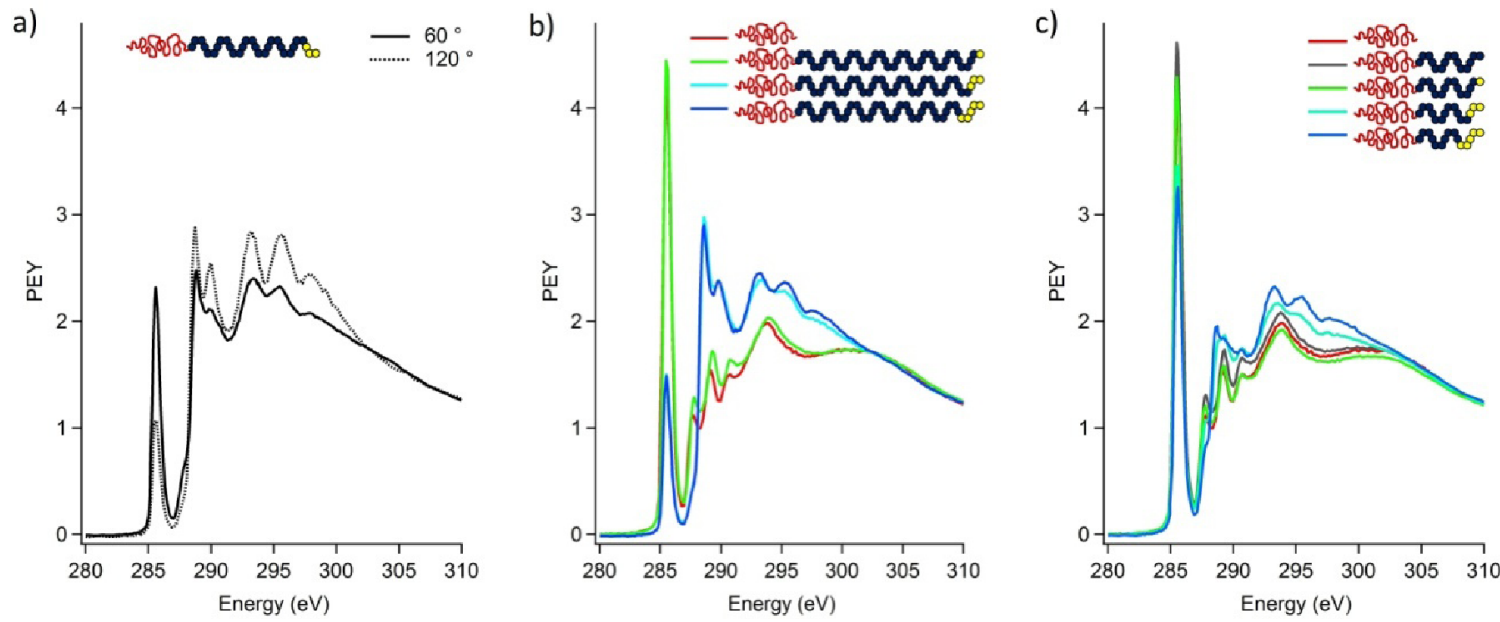

Figure 9. $\mathrm{C}$ 1s NEXAFS spectra of (a) PS- $b-\left(\mathrm{EG}_{27}-\mathrm{NF}_{3}\right)$ at different $\theta$. At a higher $\theta$, where the technique is more surface sensitive, the $\pi^{*} \mathrm{C}=\mathrm{C}$ peak at $285.5 \mathrm{eV}$ is smaller, indicating less PS and more peptoid in close proximity to the surface. (b) Block copolymers of PS with 45-mer peptoids with various numbers of fluorinated groups $\left(\theta=60^{\circ}\right.$ for all spectra). (c) Block copolymers of PS with 15-mer peptoids with various numbers of fluorinated groups $\left(\theta=60^{\circ}\right.$ for all spectra). 


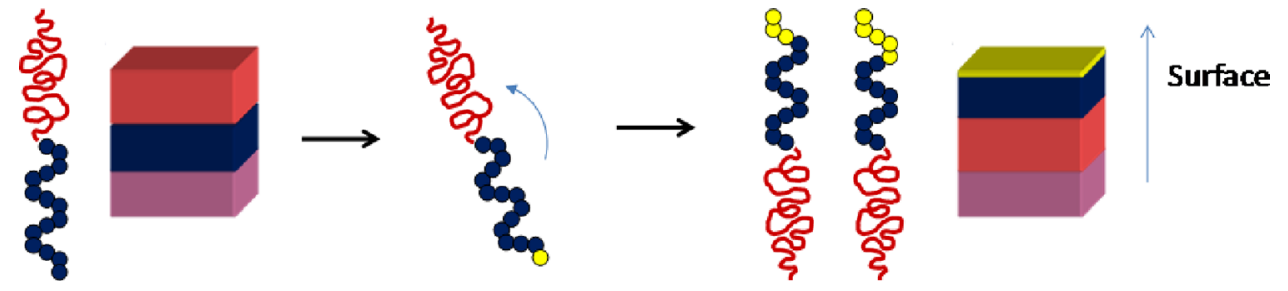

Figure 10. Schematic representation of PS- $b$-peptoid surface structures (the purple layers represent the bulk of the material). When the peptoid contains no fluorinated monomers, PS surface segregates. For one fluorinated monomer, some of the peptoid migrates to the surface; the amount depends on peptoid length. For three and five fluorinated monomers, there is a maximal expression of peptoid at the surface. The surface schematic depicts sharp boundaries between the peptoid phase and the PS. In reality, phase boundaries are blurred due to mixing of the PS with the peptoid; PS chains may even protrude through the top peptoid layer.

where $d_{\theta}$ is the fraction of surface depth that is probed $(0.41$ for $\theta=120^{\circ}$ and 0.99 for $\left.\theta=60^{\circ}\right)$ and $y_{\theta}$ is the area of the $\pi^{*}{ }_{\mathrm{C}=\mathrm{C}}$ peak. $A<1$ indicates incomplete coverage of PS by the peptoid. $A>1$ indicates that complete coverage of the surface by a layer of peptoid (such as depicted in Figure 10) may exist, although this requires the assumption of invariance of density in the $z$ direction of the film, which may not be the case. For the 15mer, 30-mer, and 45-mer block copolymers with five fluorinated groups, $A$ values are $0.54,0.89$, and 1.26, respectively. These $A$ values indicate that quite a lot of PS displays at the surface and that only a 45-mer peptoid block could potentially cover the entire surface.

Influence of Sequence. Peptoid sequence can also be used to influence the composition of the surface. In order to investigate the effect of sequence, a different series of PS- $b$ peptoid block copolymers were synthesized (Table 3, entries $13-17$ ). The molecular weight of the PS was $\sim 13.7 \mathrm{~K}$, and the peptoid was a 30-mer in which the position of the three fluorinated groups was systematically altered. As there is an entropic driving force for surface segregation of end-groups, it is not surprising that the lowest amount of surface segregated PS (lowest $\pi_{\mathrm{C}=\mathrm{C}}^{*}$ ) is detected for the end-fluorinated peptoid. Moving the fluorinated groups slightly closer to the PS block causes a big effect in the chain conformation because now loops must display at the surface, thereby decreasing the thickness of the peptoid layer (see inset in Figure 11). The effect plateaus for sequences in which the fluorinated groups are even closer to the PS, although arguably more surface phase mixing occurs in this situation because the fluorinated groups that are positioned closer to PS drag both the peptoid as well as PS to the surface.

Surface Orientation. Information on the net orientation of all molecules at roughly equal surface depth can be obtained by comparing the signals acquired at lower values of $\theta$. For all block copolymers used in this study with three or less fluorinated monomers, the NEXAFS signals at $\theta=30^{\circ}$ and $\theta$ $=60^{\circ}$ overlap, indicating no specific bond orientation. Figure 12 shows the NEXAFS spectrum of PS- $b-\left(\mathrm{EG}_{40}-\mathrm{NF}_{5}\right)$ as an example of the behavior of the peptoids containing five fluorinated monomers. At an incidence angle of $60^{\circ}$ (less grazing), the $\pi^{*}{ }_{\mathrm{C}=\mathrm{O}}$ peak and the $\sigma^{*}{ }_{\mathrm{C}-\mathrm{C}}$ peak slightly decrease, whereas the intensities of the $\sigma^{*}{ }_{\mathrm{C}-\mathrm{N}}$ and $\sigma^{*}{ }_{\mathrm{C}-\mathrm{F}}$ peaks increase. The peptoid molecules possess $\mathrm{C}-\mathrm{C}$ bonds in orthogonal directions, but most of them are located in the side chains as opposed to the backbone. The sets of bonds with decreasing intensities are thus positioned orthogonally to the sets of bonds with increasing intensities in the molecule, and the results indicate that the peptoids are somewhat oriented with their backbone perpendicular to the surface. Results at $90^{\circ}$ further corroborate these findings, although a significant change in the

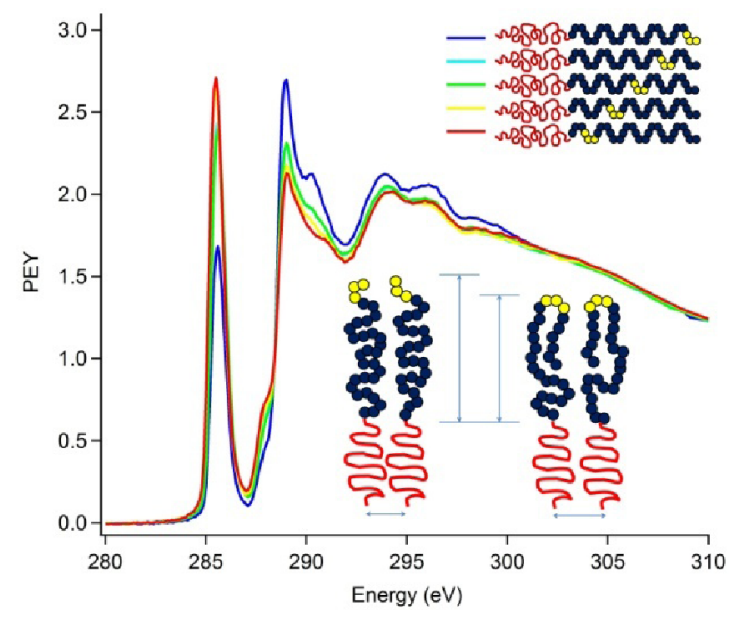

Figure 11. C 1s NEXAFS spectra of block copolymers of $13.7 \mathrm{~K}$ PS with 30-mer peptoids containing three fluorinated monomers in various sequences. The inset demonstrates the effect of surface loop formation on the peptoid layer thickness in the case of clear phase boundaries. In reality, phase boundaries blur to the point where a small fraction of PS chains protrude at the surface.

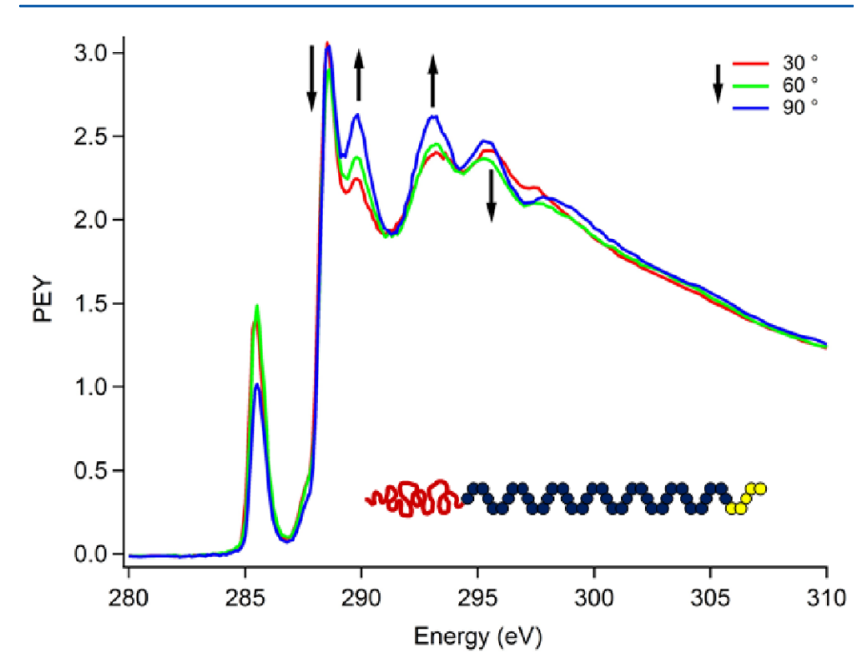

Figure 12. C 1 s NEXAFS spectrum of PS- $b-\left(E_{40}-\mathrm{NF}_{5}\right)$ at various $\theta$. Arrows indicate the decreases/increases in peak height between $\theta=$ $30^{\circ}$ and $\theta=60^{\circ}$ and to a lesser extent $\theta=90^{\circ}$.

surface sensitivity of the technique at this angle also results in a decreased PS-correlated $\pi^{*} \mathrm{C}=\mathrm{C}$ peak. Therefore, both orientational and depth-dependent information are convoluted. The observed orientation is most likely a result of the crystallinity of the fluorinated part of the peptoid, which only occurs for 
groups of five fluorinated monomers and may explain the lack of orientation in samples with less fluorinated groups.

Wet Behavior: Static Contact Angles. Contact angle measurements provide further information about the properties of the surfaces. Figure 13 shows the static contact angles for the

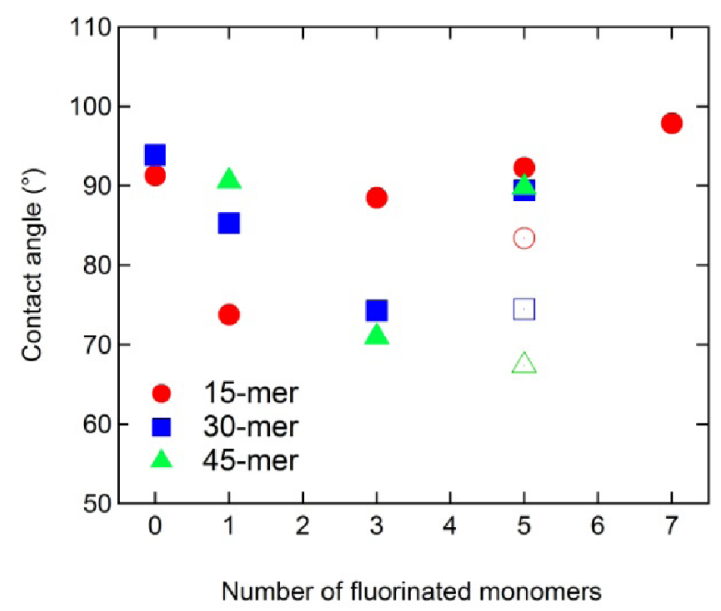

Figure 13. Static contact angles of $\mathrm{PS}_{11 \mathrm{~K}}-b$-peptoid block copolymers. Open symbols represent values taken after $24 \mathrm{~h}$ of immersion in water.

end-fluorinated peptoid series (Table 3, entries 1-12). It should be noted that all surfaces except PS- $b-\left(\mathrm{EG}_{8}-\mathrm{NF}_{7}\right)$ exhibited a low root mean square (rms) roughness as measured with AFM $(<0.5 \mathrm{~nm})$. The most highly fluorinated surface was clearly rougher $(\sim 2 \mathrm{~nm})$.

When the peptoids contain no fluorinated monomers, the surfaces basically consist of PS, and the contact angles of $\sim 90^{\circ}$ reflect that of pure PS surfaces. When one fluorinated monomer is present, a decrease in the contact angle indicates a change in surface composition (more peptoid at the surface) for all block copolymers. As seen with NEXAFS, this change depends on peptoid length. It is especially interesting to note that even though NEXAFS measurements of the 15-mer block copolymer did not clearly indicate the peptoid at the surface, the drop in contact angle does indicate a change in surface chemistry.

For three fluorinated monomers, the contact angle for the 30 - and 45 -mer block copolymers drops by $\sim 20^{\circ}$, indicating a significant change in surface chemistry, whereas for five fluorinated monomers, the contact angle increases again to that of PS. Because NEXAFS measurements indicate the same change in surface chemistry for samples with both three and five fluorinated monomers, it is likely that surface reconstruction occurs. In the case of three fluorinated monomers, surface reconstruction is fast and the contact angle mostly reflects the more hydrophilic part of the peptoid, as the fluorinated monomers likely bend away from the surface. This hypothesis is supported by the fact that the contact angles for 30- and 45-mer block copolymer samples with three fluorinated groups visually changed from a relatively round drop to the final contact angle value within seconds of depositing the drop on the surface. For five fluorinated monomers, interactions between the fluorinated chains may be strong enough to prevent rapid reconstruction, and in addition, no visual reconstruction seemed to occur. This reasoning also explains the significantly higher contact angles of the 15-mer block copolymers with higher fluorine content. In these polymers, the fraction of hydrophilic peptoid is so low that no significant reconstruction can occur.
Remeasuring the contact angle of the samples with five fluorinated groups after $24 \mathrm{~h}$ of immersion in water results in decreased contact angle values (samples were dried for $30 \mathrm{~s}$ in a stream of $\mathrm{N}_{2}$ within 5 min before remeasurement), indicating that the surfaces reconstruct on a longer time scale. It is possible that surfaces could reconstruct back to some extent in the small amount of time that they were exposed to air, but the differences between the samples before and after immersion give an indication of the behavior of the surfaces in water. It seems that the ability of the polymers to reconstruct depends on peptoid length: longer peptoids can reconstruct more readily to display the hydrophilic part of the peptoid. Remeasurement of the contact angle of other samples after water immersion resulted in roughly the same contact angles, indicating no or only slight reconstruction of these samples after exposure to water. Figure 14 schematically depicts the

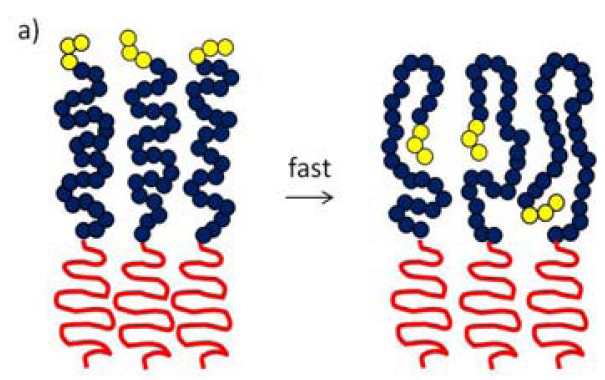

b)

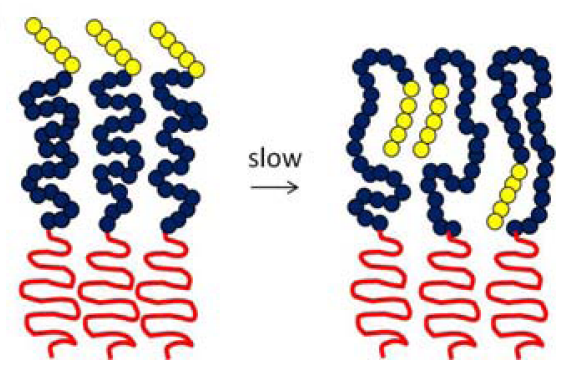

Figure 14. Schematic representation of surface reconstruction in PS- $b$ peptoid thin films. (a) For noncrystalline samples, reorganization upon contact with water is fast. (b) Reorganization for crystalline structures is slower. Note the difference in peptoid layer thickness for the reconstructed surfaces. Again, phase mixing is not depicted here.

observed effects. The proposed structures after rearrangement are supported by NEXAFS measurements of the block copolymers after immersion in water (not shown), in which a larger $\pi^{*}{ }_{C}=\mathrm{C}$ peak indicated more PS (less peptoid) in the proximity of the surface.

Wet Behavior: Dynamic Contact Angles. All block copolymer surfaces exhibited a $\sim 10^{\circ}$ contact hysteresis in dynamic contact angle measurements, and especially the reconstruction of PS- $b-\left(\mathrm{EG}_{40}-\mathrm{NF}_{5}\right)$ was clearly evident from the measurement results. Dynamic contact angles were measured using the method described in the Experimental Section. In almost all cases, the $2 \mu \mathrm{L}$ drop displayed identical behavior to that recorded in Figure 15a,b. Upon an increase of the tilting angle, the advancing contact angle initially increases before reaching a maximum, after which it slightly decreases again. The receding angle steadily decreases until it reaches its final value at a tilt of $90^{\circ}$. During tilting, both the advancing and receding contact line are pinned to the surface, and the accompanying angles respectively increase and decrease up to 

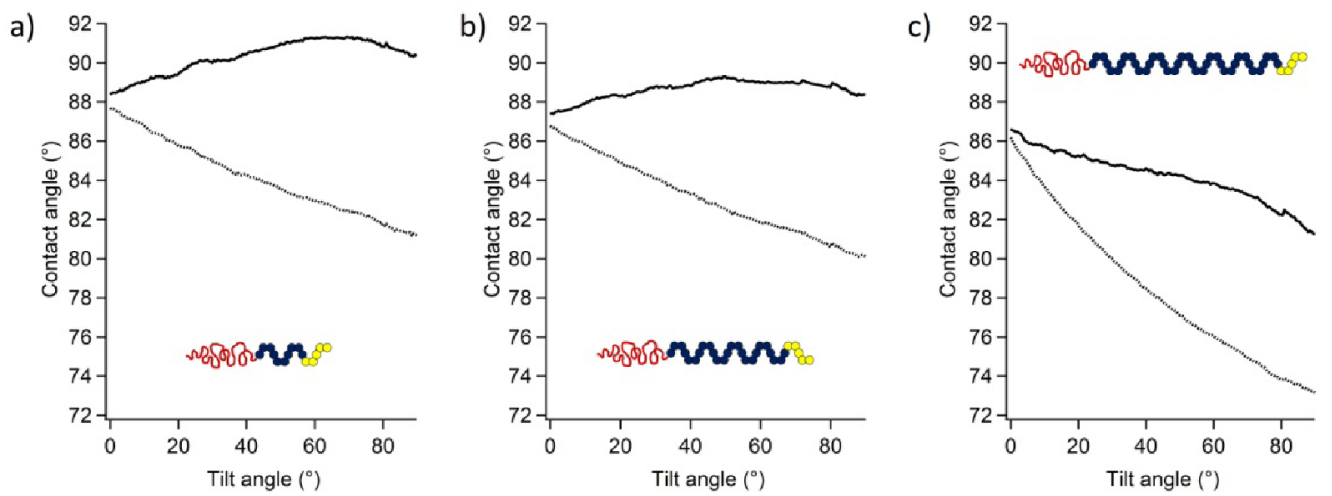

Figure 15. Dynamic contact angles of (a) $\mathrm{PS}_{11 \mathrm{~K}^{-}}-\left(\mathrm{EG}_{10^{-}}-\mathrm{NF}_{5}\right),(\mathrm{b}) \mathrm{PS}_{11 \mathrm{~K}}-b-\left(\mathrm{EG}_{25}-\mathrm{NF}_{5}\right)$, and (c) $\mathrm{PS}_{11 \mathrm{~K}^{-}}-b-\left(\mathrm{EG}_{40}-\mathrm{NF}_{5}\right)$; solid lines represent advancing contact angles, and dotted lines represent receding contact angles.

the point where the drop starts to move down the surface due to gravity. Because of the amphiphilicity of the surfaces, the receding part of the droplet stays pinned while the front end is already moving, causing the drop to elongate. This elongation results in a higher force pinning the drop to the surface, and because of the low volume of the drop, its movement slows down before it comes to a complete halt. One would expect both the advancing and receding angles to then remain constant; however, evaporation of the small drop likely leads to a decrease in both angles from this point onward. Careful modeling and experiments with different drop sizes and tilting speeds to unravel the effect of gravity, evaporation, and contact angle hysteresis could determine the exact points along the graph at which the different events take place, but for characterization of the surface, the contact angle hysteresis is the most important. This hysteresis remains constant after the maximum of the advancing contact angle (both angles decrease with approximately the same rate after this maximum, $65^{\circ}$ tilt and onward in Figure 15a,b). For all samples, the advancing angle lay within $3^{\circ}$ above the static contact angle, and the hysteresis was $10 \pm 2^{\circ}$.

The dynamic contact angle of PS- $b-\left(\mathrm{EG}_{40}-\mathrm{NF}_{5}\right)$ behaves differently from all others, although the contact angle hysteresis at the end of the measurement is similar $\left(9^{\circ}\right)$ and the slope of the advancing angle graph also contains an inflection point (Figure 15c). The contact angle for this sample steadily decreases from the start of the measurement, and the decrease of the receding contact angle has a steeper slope. Both are signs that reconstruction of this surface is taking place during the measurement. We hypothesize that molecules at the advancing contact line are reorganizing to display the more hydrophilic part of the peptoid as the stage is tilted, resulting in no obvious increase in contact angle even though gravity is pulling the drop downward. Apparently, reorganization of these surfaces occurs on the time scale of the measurement, whereas reorganization of surfaces with five fluorinated groups and shorter hydrophilic sequences is slower due to less conformational freedom of the chains. Future experiments with varying drop sizes and tilt speeds can further elucidate this effect.

\section{CONCLUSION}

We demonstrated tunable surface properties using sequencespecific polypeptoids by systematically tuning the amount and sequence of hydrophobic monomers in a predominantly hydrophilic peptoid chain in PS- $b$-peptoid block copolymers. Three fluorinated groups in peptoid sequences of up to 45 hydrophilic monomers were needed to lower the surface energy of the peptoid enough for maximal surface segregation of the peptoid to occur. The amount of peptoid displayed at the surface was controlled by changing either the total length of the peptoid or the position of the fluorinated monomers within the peptoid. The latter method controls the thickness of the peptoid surface layer by changing chain conformation and creating loops at the surface.

Although peptoid presentation at the surface reached a maximum for three fluorinated groups, the wetting behavior of those peptoids and peptoids containing five fluorinated monomers was strikingly different. Surface reconstruction of peptoid chains that contain a sequence of only three fluorinated monomers and up to 42 hydrophilic monomers occurred within seconds, whereas reorganization of surfaces containing five fluorinated monomers was an order of magnitude slower. This effect is likely caused by stronger interactions between the fluorinated groups. In the case of the longest peptoid block, dynamic contact angle measurements using an automated tilting base were able to capture the surface reorganization of the peptoid in real time.

Overall, polypeptoids were shown to be a versatile platform for studying sequence dependent surface properties of block copolymers, which can be important for elucidating surface reconstruction effects in e.g. antifouling coatings. Although most effects demonstrated in this paper were easily explained by simple qualitative arguments, this study demonstrates the potential of polypeptoids to play an important role as model systems in future studies where exact control over polymer sequence and length is important. Sequence-specific polymers play a key role in nature, and by analogy, it is to be expected that synthetic sequence-specific polymers will play an important role in applied materials science as well.

\section{AUTHOR INFORMATION}

\section{Corresponding Author}

*E-mail: Segalman@berkeley.edu.

\section{Notes}

The authors declare no competing financial interest.

\section{ACKNOWLEDGMENTS}

This work was supported by the Office of Naval Research (ONR) in the form of a Presidential Early Career Award (PECASE) in Science and Engineering for R.A.S. W.v.Z. gratefully acknowledges The Netherlands Organization for Scientific Research (NWO) for a Rubicon fellowship. 
Polypeptoid synthesis and associated chemical characterization were performed at the Molecular Foundry, a Lawrence Berkeley National Laboratory user facility supported by the Office of Science, Office of Basic Energy Sciences, U.S. Department of Energy, under Contract DE-AC0205CH11231. Use of the National Synchrotron Light Source, Brookhaven National Laboratory, was supported by the U.S. Department of Energy, Office of Science, Office of Basic Energy Sciences, under Contract DE-AC02-98CH10886. The authors thank Ed Kramer and Mike Dimitriou (University of California, Santa Barbara) for their help in acquiring and analyzing NEXAFS results.

\section{REFERENCES}

(1) Binder, K.; Muller, M.; Vink, R. L. C. Macromol. Theory Simul. 2011, 20, 600 .

(2) Bates, F. S.; Fredrickson, G. H. Phys. Today 1999, 52, 32.

(3) Park, C.; Yoon, J.; Thomas, E. L. Polymer 2003, 44, 6725.

(4) Krishnan, S.; Weinman, C. J.; Ober, C. K. J. Mater. Chem. 2008, $18,3405$.

(5) Grozea, C. M.; Walker, G. C. Soft Matter 2009, 5, 4088.

(6) Cho, Y. J.; Sundaram, H. S.; Weinman, C. J.; Paik, M. Y.; Dimitriou, M. D.; Finlay, J. A.; Callow, M. E.; Callow, J. A.; Kramer, E. J.; Ober, C. K. Macromolecules 2011, 44, 4783.

(7) Gudipati, C. S.; Finlay, J. A.; Callow, J. A.; Callow, M. E.; Wooley, K. L. Langmuir 2005, 21, 3044.

(8) Sundaram, H. S.; Cho, Y. J.; Dimitriou, M. D.; Weinman, C. J.; Finlay, J. A.; Cone, G.; Callow, M. E.; Callow, J. A.; Kramer, E. J.; Ober, C. K. Biofouling 2011, 27, 589.

(9) Park, D.; Weinman, C. J.; Finlay, J. A.; Fletcher, B. R.; Paik, M. Y.; Sundaram, H. S.; Dimitriou, M. D.; Sohn, K. E.; Callow, M. E.; Callow, J. A.; Handlin, D. L.; Willis, C. L.; Fischer, D. A.; Kramer, E. J.; Ober, C. K. Langmuir 2010, 26, 9772.

(10) Krishnan, S.; Ayothi, R.; Hexemer, A.; Finlay, J. A.; Sohn, K. E.; Perry, R.; Ober, C. K.; Kramer, E. J.; Callow, M. E.; Callow, J. A.; Fischer, D. A. Langmuir 2006, 22, 5075.

(11) Zuckermann, R. N.; Kerr, J. M.; Kent, S. B. H.; Moos, W. H. J. Am. Chem. Soc. 1992, 114, 10646.

(12) Simon, R. J.; Kania, R. S.; Zuckermann, R. N.; Huebner, V. D.; Jewell, D. A.; Banville, S.; Ng, S.; Wang, L.; Rosenberg, S.; Marlowe, C. K.; Spellmeyer, D. C.; Tan, R. Y.; Frankel, A. D.; Santi, D. V.; Cohen, F. E.; Bartlett, P. A. Proc. Natl. Acad. Sci. U. S. A. 1992, 89, 9367.

(13) Miller, S. M.; Simon, R. J.; Ng, S.; Zuckermann, R. N.; Kerr, J. M.; Moos, W. H. Drug Dev. Res. 1995, 35, 20.

(14) Zuckermann, R. N.; Kodadek, T. Curr. Opin. Mol. Ther. 2009, 11, 299.

(15) Zuckermann, R. N. Biopolymers 2011, 96, 545.

(16) Fowler, S. A.; Blackwell, H. E. Org. Biomol. Chem. 2009, 7, 1508.

(17) Rosales, A. M.; Murnen, H. K.; Zuckermann, R. N.; Segalman, R. A. Macromolecules 2010, 43, 5627.

(18) Murnen, H. K.; Rosales, A. M.; Jaworsk, J. N.; Segalman, R. A.; Zuckermann, R. N. J. Am. Chem. Soc. 2010, 132, 16112.

(19) Nam, K. T.; Shelby, S. A.; Choi, P. H.; Marciel, A. B.; Chen, R.; Tan, L.; Chu, T. K.; Mesch, R. A.; Lee, B. C.; Connolly, M. D.; Kisielowski, C.; Zuckermann, R. N. Nat. Mater. 2010, 9, 454.

(20) Chen, C. L.; Qi, J. H.; Zuckermann, R. N.; DeYoreo, J. J. J. Am. Chem. Soc. 2011, 133, 5214.

(21) Guo, L.; Zhang, D. H. J. Am. Chem. Soc. 2009, 131, 18072.

(22) Jones, R. A. L. J. Polym. Sci., Polym. Phys. 2005, 43, 3367.

(23) Barron, A. E.; Zuckermann, R. N. Curr. Opin. Chem. Biol. 1999, 3,681 .

(24) Bothe, M.; Schmidt-Naake, G. Macromol. Rapid Commun. 2003, 24, 609 .

(25) Figliozzi, G. M.; Goldsmith, R.; Ng, S. C.; Banville, S. C.; Zuckermann, R. N. Method. Enzymol. 1996, 267, 437.

(26) Rosales, A. M.; McCulloch, B. L.; Zuckermann, R. N.; Segalman, R. A. Macromolecules 2012, DOI: $10.1021 / \mathrm{ma3} 00625 \mathrm{~b}$.
(27) Holub, J. M.; Jang, H. J.; Kirshenbaum, K. Chemistry 2006, 4, 1497.

(28) Genzer, J.; Sivaniah, E.; Kramer, E. J.; Wang, J. G.; Korner, H.; Char, K.; Ober, C. K.; DeKoven, B. M.; Bubeck, R. A.; Fischer, D. A.; Sambasivan, S. Langmuir 2000, 16, 1993.

(29) Paik, M. Y.; Krishnan, S.; You, F. X.; Li, X. F.; Hexemer, A.; Ando, Y.; Kang, S. H.; Fischer, D. A.; Kramer, E. J.; Ober, C. K. Langmuir 2007, 23, 5110.

(30) Sohn, K. E.; Dimitriou, M. D.; Genzer, J.; Fischer, D. A.; Hawker, C. J.; Kramer, E. J. Langmuir 2009, 25, 6341.

(31) Samant, M. G.; Stohr, J.; Brown, H. R.; Russell, T. P.; Sands, J. M.; Kumar, S. K. Macromolecules 1996, 29, 8334.

(32) Liu, Y.; Russell, T. P.; Samant, M. G.; Stohr, J.; Brown, H. R.; Cossy-Favre, A.; Diaz, J. Macromolecules 1997, 30, 7768.

(33) Kudirka, R.; Tran, H.; Sanii, B.; Nam, K. T.; Choi, P. H.; Venkateswaran, N.; Chen, R.; Whitelam, S.; Zuckermann, R. N. Biopolymers 2011, 96, 586.

(34) Stöhr, J. NEXAFS Spectroscopy, 1st ed.; Springer: Berlin, 2003; p $\mathrm{xv}, 403 \mathrm{pp}$.

(35) Genzer, J.; Kramer, E. J.; Fischer, D. A. J. Appl. Phys. 2002, 92, 7070.

(36) Bunn, C. W.; Howells, E. R. Nature 1954, 174, 549. 